\title{
GENERAL INEQUALITIES FOR MULTIPOINT PADÉ APPROXIMANTS TO A STIELTJES FUNCTION EXPANDED AT REAL POINTS
}

\author{
S. TOKARZEWSKI AND E. WAJNRYB
}

\begin{abstract}
In this paper we establish the general inequalities for diagonal and subdiagonal multipoint Padé approximants to a Stieltjes function $f$ in terms of power expansion of $f$ on the real line. The inequalities derived produce the best upper and lower bounds on $f$ with respect to the given coefficients of Stieltjes series. As an example of applications sequences of upper and lower Padé bounds converging to the effective dielectric constant of a random array of spheres are evaluated.
\end{abstract}

Mathematics subject classification (2010): 11J70, 41A21.

Keywords and phrases: $N$-point Padé approximants, Stieltjes functions, continued fractions.

\section{REFERENCES}

[1] G. BAKer, JR, Essentials of Padé Approximants, Academic Press, New York, USA, 1975.

[2] G. A. BAKer, JR AND P. Graves-Morris, Padé Approximants, volume 59 of Encyclopedia of Mathematics and its Applications, Cambridge University Press, London, second edition, 1996.

[3] M. BARnsley, The bounding properties of the multipoint Padé approximant to a series of Stieltjes on the real line, J. Math. Phys., 14, 3 (1973).

[4] A. Bultheel, P. Gonzáles-Vera, E. Hendricksen, and O. Nuastad, Monotonicity of multipoint padé approximants, Preprint of paper presented at ICRA99, pages 1-12, 1999. http://citeseerx.ist.psu.edu/viewdoc/summary?doi=10.1.1.43.7800.

[5] A. Bultheel, P. Gonzáles-Vera, E. Hendriksen, and O. NJastad, Orthogonal rational functions on the real half line with poles in [-infinity, zero]. J. Comput. Appl. Math., 179 (2005), $121-151$.

[6] A. Bultheel, P. Gonzáles-Vera, And R. Orive, Quadrature on the half-line and two-point Padé approximants to Stieltjes functions, Part I, Algebraic aspects, J. of Comp. Appl. Math., 65 (1995), 57-72.

[7] B. Cichocki AND B. U. Felderhof, Electrostatic spectrum and dielectric constant of nonpolar hard sphere fluids, J. Chem. Phys., 90, 9 (May 1989), 4960-496.

[8] B. Cichocki and B. U. Felderhof, Cavity field and reaction in nonpolar fluids, J. Chem. Phys., 92, 10 (May 19920), 6104-6111.

[9] J. Gilewicz And A. P. Magnus, Optimal inequalities of Padé approximants errors in the Stieltjes case: Closing result, Integral Transform. Spec. Funct., 1 (1993), 9-18.

[10] K. Golden AND G. PAPANICOLAOU, Bounds for effective parameters of heterogeneous media by analytic continuation, Comm. Math. Phys., 90, 4 (1983), 473-491.

[11] K. Hinsen ANd B. Felderhof, Dielectric constant of a suspension of uniform spheres, Physical Review B, 46, 20 (November 1922), 12955-12963.

[12] S. TokARZEWSKI, $N$-point Padé approximants to real valued Stieltjes series with nonzero radii of convergence, J. Comp. Appl. Math., 75 (1996), 259-280.

[13] S. TokARZEWSKI, Two- point Padé approximants for the expansion of Stieltjes function in a real domain, J. Comp. Appl. Math., 67 (1996), 59-72.

[14] S. TokARZEWs Ki, A. Magnus, AND J. Gilewicz, Estimation of a Stieltjes function expanded to Taylor series at complex conjugate points, J. Comp. Appl. Math., 233, 3 (2009), 835-841. 
[15] S. Tokarzews Ki AND J. J. Telega, Bounds on effective moduli by analytical continuation of the Stieltjes function expanded at zero and infinity, Z. angew. Math. Phys., 48 (1997), 1-20. 